\title{
THE SPACE OF MINIMAL PRIME IDEALS OF $C(X)$ NEED NOT BE BASICALLY DISCONNECTED
}

\author{
A. DOW, M. HENRIKSEN, RALPH KOPPERMAN AND J. VERMEER
}

(Communicated by Doug W. Curtis)

\begin{abstract}
Problems posed twenty and twenty-five years ago by M. Henriksen and $M$. Jerison are solved by showing that the space of minimal prime ideals of the ring $C(X)$ of continuous real-valued functions on a compact (Hausdorff) space need not be basically disconnected-or even an $F$-space.
\end{abstract}

If $R$ is a commutative ring, let $\operatorname{Spec}(R)=S(R)$ denote the set of prime ideals of $R, \operatorname{Minspec}(R)=m(R)$ the subset of minimal elements of $S(R)$, and if $R$ has an identity element $\operatorname{Maxspec}(R)=M(R)$, the set of maximal elements of $S(R)$. We impose the hull kernel or Zariski topology on $S(R)$; that is the topology with base $\left\{h^{c}(a): a \in R\right\}$, where $h^{c}(a)=\{P \in S(R): a \notin P\}$, and we regard $m(R)$ and $M(R)$ as subspaces of $S(R)$. For background, see [Ho, $\mathbf{H J}$, and $\mathbf{K}$ ].

Below, $X$ will always denote a Tychonoff space. We are concerned particularly with $m(R)$ in case $R=C(X)$, the ring of all real-valued, continuous functions on $X$, and our aim is to present a solution to a problem posed by M. Henriksen and M. Jerison about $m(C(X))$ in 1961 and 1965; see [HJ]. Terms not defined explicitly below may be found in [GJ].

For $f \in C(X)$, let $Z(f)=\{x: f(x)=0\}, \operatorname{coz}(f)=X-Z(f), \operatorname{spt}(f)=$ $\mathrm{Cl}(\operatorname{coz}(f))$, and zero (cozero) sets [supports] are sets of the form $Z(f)(\operatorname{coz}(f))$ $[\operatorname{spt}(f)]$ for some $f \in C(X)$. If $x \in X, M_{x}=\{f \in C(X): x \in Z(f)\}$ and $O_{x}=$ $\{f \in C(X): x \in \operatorname{Int}(Z(f))\}$. $X$ is called an $F$-space if each $P \in S(C(X))$ contains a unique $P^{\prime} \in m(C(X))$. It is well known (see [GJ]) that $M(C(X))=\left\{M_{x}: x \in X\right\}$ for arbitrary compact Hausdorff $X$, and that $m(C(X))=\left\{O_{x}: x \in X\right\}$ if $X$ is also an $F$-space.

A pair $A, B$ of subsets of $X$ are said to be completely separated if there is an $f \in C(X)$ such that $f[A]=\{0\}$ and $f[B]=\{1\}$. Completely separated subsets have disjoint closures, and the converse holds if $X$ is a normal space.

$X$ is basically (extremally) disconnected if whenever $A$ is an open set and $B$ a cozero (open) set disjoint from $A$, then $A$ and $B$ are completely separated. As shown in $[\mathbf{G J}], X$ is basically (extremally) disconnected iff the closure of each cozero set (open set) is open. Thus every extremally disconnected space is basically disconnected. It is also shown in [GJ] that $X$ is an $F$-space iff disjoint cozero sets are completely separated, so basically disconnected spaces are all $F$-spaces. $X$ is called an $F^{\prime}$-space if disjoint cozero sets have disjoint closures. Every $F$-space is an $F^{\prime}$-space but the converse may fail if $X$ is nonnormal [GH].

Received by the editors October 22, 1986 and, in revised form, September 23, 1987.

1980 Mathematics Subject Classification (1985 Revision). Primary 54C40, 06F25.

(C) 1988 American Mathematical Society $0002-9939 / 88 \$ 1.00+\$ .25$ per page 
As usual we let $\beta X$ denote the Stone-Cech compactification of $X$ and $X^{*}$ denote $\beta X-X$. We let $\mathbf{N}$ denote the countably infinite discrete space. The following are established in $[\mathbf{H J}]$ :

(a) $m(C(X))$ is countably compact (we have shown in [DHKV] that the closure of each weakly Lindelöf subset is compact),

(b) $m(C(X))$ is basically disconnected if it is locally compact,

(c) $m\left(C\left(\mathbf{N}^{*}\right)\right)$ is nowhere locally compact,

(d) it follows easily from $[\mathbf{H J}], 4.2$ that the closure of a countable union of basic open sets (i.e., sets of the form $h^{c}(a)$ ) is a basic open set.

M. Henriksen and M. Jerison asked in 1961 if $m\left(C\left(\mathbf{N}^{*}\right)\right)$ is basically disconnected, and in 1965 they asked whether for any $X, m(C(X))$ fails to be basically disconnected; see $[\mathbf{H J}]$. By condition (d) these seem natural questions. Below we answer the second question in the affirmative (in ZFC), and the first in the negative if Martin's Axiom (MA) holds; in particular, if the continuum hypothesis ( $\mathrm{CH}$ ) holds. For a statement of Martin's Axiom see $[\mathbf{K u}]$.

A key part of the solution is the notion of a $P$-set: it is a nonempty compact subset $K$ of $X$ for which every countable intersection of neighborhoods of $K$ is a neighborhood of $K$. Equivalently, $K$ is a $P$-set iff it is nonempty, compact and completely separated from any cozero set disjoint from it. Clearly if a zero set is a $P$-set, then it is open. Many facts about $P$-sets are given in [vM].

A continuous surjection $g: Y \rightarrow Z$ is called irreducible or said to map $Y$ irreducibly onto $Z$ if no proper closed subset of $Y$ is mapped onto $Z$ by $g$. For every compact $Y$, there is a unique extremally disconnected space $E Y$ minimal with respect to having an irreducible map onto $X$, which we call the absolute of the space $Y$. It is the Stone space of the Boolean algebra of regular closed subsets of $X$; for background see [Wo].

The proof of the following folk-lemma is an exercise.

1. LEMMA. If $g: Y \rightarrow Z$ is closed and irreducible and $D$ is dense in $Z$ then $f^{-1}[D]$ is dense in $Y$.

As noted above, if $X$ is an $F$-space then $m(C(X))=\left\{O_{x}: x \in X\right\}$. Thus $x \rightarrow O_{x}$ naturally identifies $X$ with $m(C(X))$.

2. LEMMA. If $X$ is a compact $F$-space then the map $x \rightarrow O_{x}$ is a homeomorphism from $X$ with the topology whose base is $\{\operatorname{spt}(f): f \in C(X)\}$ to $m(C(X))$ with the hull-kernel topology.

Proof. For $f \in C(X), h^{c}(f)=\left\{O_{x}: f \notin O_{x}\right\}=\left\{O_{x}: x \notin \operatorname{Int}(Z(f))\right\}=$ $\left\{O_{x}: x \in \operatorname{spt}(f)\right\}$, and as noted above, $\left\{h^{c}(f): f \in C(X)\right\}$ is a base for the hullkernel topology on $m(C(X))$.

From this characterization of the topology of $m(C(X))$ when $X$ is a compact $F$-space we obtain:

3. THEOREM. If $X$ is a compact $F$-space in which every zero set is regular closed, and if $X$ contains a $P$-set $E$ which maps irreducibly onto $[0,1]$, then $m(C(X))$ is not an $F^{\prime}$-space. In particular, $m(C(X))$ is not basically disconnected.

Proof. Suppose $p: E \rightarrow[0,1]$ is irreducible. Let $A=\left\{a_{n}: n \in \mathbf{N}\right\}, B=$ $\left\{b_{n}: n \in \mathbf{N}\right\}$ be two disjoint, countably infinite dense subsets of $[0,1]$. By Lemma 
$1, p^{-1}[A]$ and $p^{-1}[B]$ are disjoint dense subsets of $E$. Since $E$ is compact, $p$ has a continuous extension $g: X \rightarrow[0,1]$. For each positive integer $n$, both $g^{-1}\left(a_{n}\right)$ and $g^{-1}\left(b_{n}\right)$ are zero sets and by assumption, each has dense interior. By Lemma $2, \operatorname{Int}\left(g^{-1}\left(a_{n}\right)\right)$ is open, as well as closed, in the hull-kernel topology, so $L(A)=$ $\bigcup\left\{\operatorname{Int}\left(g^{-1}\left(a_{n}\right)\right): n \in \mathbf{N}\right\}, L(B)=\bigcup\left\{\operatorname{Int}\left(g^{-1}\left(b_{n}\right)\right): n \in \mathbf{N}\right\}$, are nonempty cozero sets of $m(C(X))$, which are clearly disjoint. Our theorem will be proved once we establish that $E$ is contained in the hull-kernel closure of both $L(A)$ and $L(B)$.

Suppose $x \in E$. By Lemma 2, each basic neighborhood of $x$ takes the form $\mathrm{Cl}_{X}(U)$, where $U=\operatorname{coz}(h)$ for some $h \in C(X)$. Since $E$ is a $P$-set and $\mathrm{Cl}_{X}(U)$ meets $E$, we know that $U$ meets $E$. Since $p^{-1}[A]$ and $p^{-1}[B]$ are dense in $E, U$ meets each of them and hence meets both $g^{-1}[A]$ and $g^{-1}[B]$. Since $g^{-1}[A], g^{-1}[B]$ are unions of regular closed zero sets, it follows that $U$ is not disjoint from either $L(A)$ or $L(B)$. Thus $x$ is in the closure of both $L(A)$ and $L(B)$, so $m(C(X))$ is not an $F^{\prime}$-space.

K. Kunen has shown that if MA holds then $\mathbf{N}^{*}$ contains a $P$-set homeomorphic to the absolute of $[0,1]$ (see $\left[\mathbf{K u}\right.$, Theorem 1.2]). It is well known that $\mathbf{N}^{*}$ is an $F$-space in which every nonempty zero set has nonempty interior [vM, 1.6.2]. Thus the following corollary follows from Theorem 3.

4. Corollary (MA). $m\left(C\left(\mathbf{N}^{*}\right)\right)$ is not an $F$-space.

There is also a compact $X$ such that $m(C(X))$ is not an $F$-space whose existence does not depend on MA. To produce it we will need to prove the following.

5. LEMMA. If $Y$ is a zero set of $\mathrm{N}^{*}$ with nonempty boundary, then that boundary is a $P$-set of $Y$.

ProOF. Let $Y=Z(f), f \in C(X)$. By duality it will suffice to show that the union of a sequence $\left\{S_{n}: n \in \mathbf{N}\right\}$ of closed subsets of $\operatorname{Int}(Y)$ has closure contained in $\operatorname{Int}(Y)$. Construct successive clopen subsets $U_{1}, \ldots$ such that $U_{1}$ is empty and for each $n, S_{n} \cup U_{n} \subset U_{n+1} \subset \operatorname{Int}(Y)$. Next for each $n$ let $V_{n}$ be clopen in $\mathbf{N}^{*}$ such that $f^{-1}[[0,1 /(2 n+1)]] \subset V_{n} \subset f^{-1}[[0,1 / 2 n)]$. Then $\left\{U_{n}\right\}$ and $\left\{V_{n}\right\}$ are sequences of clopen subsets of $\mathrm{N}^{*}$ such that $S_{n} \subset U_{n} \subset U_{n+1} \subset V_{m+1} \subset V_{m}$ and $\operatorname{Bd}(Y) \subset V_{m}$ for positive integers $m, n$. By [W, Chapter 3], there is a clopen subset $W$ of $\mathbf{N}^{*}$ such that $U_{n} \subset W \subset V_{m}$ for all $m, n$; thus

$$
\mathrm{Cl}\left(\bigcup\left\{U_{n}: n \in \mathbf{N}\right\}\right) \subset W \subset \bigcap\left\{V_{n}: n \in \mathbf{N}\right\}=Y,
$$

and since $W$ is open, $\operatorname{Cl}\left(\bigcup\left\{U_{n}: n \in \mathbf{N}\right\}\right) \subset \operatorname{Int}(Y)$.

Suppose $S$ and $T$ are spaces, $A$ is a closed subspace of $S$ and $f: A \rightarrow T$ is continuous. Recall that $S \cup_{f} T$ is the quotient space of the disjoint union of $S$ and $T$ obtained by identifying each $a \in A$ with $f(a) \in T$.

6. COROLLARY. There is a zero set $Z$ of $\mathbf{N}^{*}$ with a quotient space $X$ such that $m(C(X))$ is not an $F$-space.

ProOF. Let $Z$ be a zero set of $\mathbf{N}^{*}$ with nonempty boundary. It is well known that the boundary of $Z$ maps continuously onto $\beta \mathrm{N}$, hence onto $E[0,1]$. Let $f$ be such a continuous map of $Z$ onto $E[0,1]$. Since $\operatorname{Bd}(Z)$ is a nowhere dense $P$-set of $Z$ by Lemma 5 , the space $X=Z \cup_{f} E[0,1]$ is an $F$-space in which by [vM, 1.4.1 and 1.4.2] every zero set is a regular closed set. In $X$ there is a copy of $E[0,1]$ 
that is a $P$-set, so the hypotheses of Theorem 3 are satisfied, and we conclude that $m(C(X))$ fails to be an $F$-space.

7. Remarks and open problems. To apply Theorem 3 to the space $\mathbf{N}^{*}$ seems to require the existence of a separable infinite $P$-set in $\mathbf{N}^{*}$. As is noted in $\left[\mathbf{v M}\right.$, problem 6] it is an open problem whether there is such a $P$-set in $\mathbf{N}^{*}$ unless MA or some set-theoretic axiom beyond ZFC holds. Hence the question of whether $m\left(C\left(\mathbf{N}^{*}\right)\right)$ is an $F$-space remains open in ZFC.

Among other questions which come to mind from the examples given above: Suppose $X$ is an $F$-space in which zero sets are regular closed, and suppose $X$ fails to contain an infinite $P$-set. Must $m(C(X))$ be basically disconnected, or even an $F^{\prime}$-space? Is there a compact $X$ such that $m(C(X))$ is basically disconnected but not locally compact? Exactly when is $m(C(X))$ basically disconnected?

\section{REFERENCES}

[DHKV] A. Dow, M. Henriksen, R. Kopperman and J. Vermeer, The countable annihilator condition and weakly Lindelö subspaces of minimal prime ideals (submitted).

[GH] L. Gillman and M. Henriksen, Rings of continuous functions inn which finitely generated ideals are principal, Trans. Amer. Math. Soc. 82 (1956), 366-391.

[GJ] L. Gillman and M. Jerison, Rings of continuous functions, Van Nostrand, Princeton, N. J., 1960.

[HJ] M. Henriksen and M. Jerison, The space of minimal prime ideals of a commutative ring, General Topology and its Relations to Modern Analysis and Algebra (Prague, 1962), and Trans. Amer. Math. Soc. 115 (1965), 110-130.

[Ho] M. Hochster, Prime ideal structure in commutative rings, Trans. Amer. Math. Soc. 142 (1969), 43-60.

[K] J. Kist, Minimal prime ideals in commutative semigroups, Proc. London Math. Soc. (3) 13 (1963), 31-50.

[Ku] K. Kunen, Some points in $\beta \mathbf{N}$, Math. Proc. Cambridge Philos. Soc. 80 (1976), 385-398.

[vM] J. van Mill, An introduction to $\beta \mathrm{N}$, Handbook of Set-Theoretic Topology, North-Holland, Amsterdam, 1984.

[W] R. Walker, The Stone-Cech compactification, Springer-Verlag, Berlin and New York, 1974.

[Wo] R. G. Woods, A survey of absolutes of topological spaces, Topological Structures II, Mathematical Centre Tracts, no. 116, 1979, pp. 323-362.

Department of Mathematics, York University, Downsview, Ontario, CanaDA M3J 1P3

Department of Mathematics, Harvey Mudd College, Claremont, CaliforNIA 91711

Department of Mathematics, Wesleyan University, Middletown, ConnectiCUT 06457 10031

Department of Mathematics, CUNY-City College, New York, New York

Department of Mathematics, University of Toledo, Toledo, Ohio 43606 